Revista de Economia Política, vol. 42, $n^{\circ}$ 1, pp. 25-47, janeiro-março/2022

\title{
Distribuição de renda e regime de crescimento econômico no Brasil: avaliação e propostas
}

\author{
Income distribution and economic growth \\ regime in Brazil: evaluation and propositions
}

\author{
LAÍS FERNANDA DE AZEVEDO* \\ PEDRO CEZAR DUTRA FONSECA** \\ FABRICIO J. MISSIO***
}

\begin{abstract}
RESUMO: O objetivo do artigo é analisar o regime de crescimento brasileiro no período 2000-2015, levando-se em consideração a distribuição pessoal e funcional da renda. Em termos teóricos, seguimos o modelo neokaleckiano aumentado proposto por Palley (2016). Em termos empíricos, utiliza-se a metodologia de vetores autorregressivos. Os resultados mostram os efeitos positivos da redução da desigualdade em termos dos rendimentos do trabalho e da desigualdade interpessoal da renda sobre a acumulação de capital e a demanda agregada. A partir dos resultados, argumentamos em prol da adoção de um modelo de crescimento que seja capaz de conciliar distribuição de renda e políticas de incentivo às exportações.
\end{abstract}

PALAVRAS-CHAVE: Regimes de crescimento; distribuição de renda e crescimento econômico no Brasil.

ABSTRACT: The aim of the article is to empirically analyze the economic growth regimes in Brazil from 2000 to 2015. In theoretical terms, we follow the augmented neo-Kaleckian model proposed by Palley (2016). Therefore, we incorporate in the analysis the role of the personal and functional distribution of income. In empirical terms, we use a vector autoregressive model. The results suggest that the growth regime of the Brazilian economy was wage-led. This means that capital accumulation and aggregate demand were favored by policies to reduce inequality in the labor market and personal income. Based on the results,

\footnotetext{
* Professora Efetiva da Universidade Estadual de Mato Grosso do Sul (UEMS), Dourados, Mato Grosso do Sul - MS, Brasil. E-mail: laisf_azevedo@hotmail.com. Orcid: https://orcid.org/0000-0002-3746-2765.

** Professor Titular do Departamento de Economia e Relações Internacionais e do Programa de Pós-Graduação de Economia da Universidade Federal do Rio Grande do Sul (UFRGS), Porto Alegre - RS, Brasil. Pesquisador CNPQ. E-mail: pedro.fonseca@ufrgs.br. Orcid: https://orcid.org/0000-0002-3814-9578. $* * *$ Professor Adjunto do Centro de Desenvolvimento e Planejamento Regional, Universidade Federal de Minas Gerais - CEDEPLAR/UFMG, Belo Horizonte - MG, Brasil. E-mail: fjmissio@cedeplar.ufmg. br. Orcid: https://orcid.org/0000-0003-4561-6039. Submetido: 29/Outubro/2020; Aprovado: 2/Dezembro/2020.
} 
we discussed the Brazilian model, arguing in favor of adopting a growth model that can reconcile income distribution and export incentive policies.

KEYWORDS: Growth regime; income distribution and economic growth in Brazil. JEL Classification: C22; D31; D33; E25.

\section{INTRODUÇÃO}

Desde meados da década de 1990, países da América Latina engajaram-se na tentativa de impulsionar o crescimento econômico com políticas de distribuição de renda. No Brasil, destacaram-se as políticas de cunho social associadas, por exemplo, à transferência condicionada de renda, ao aumento das taxas reais de crescimento do salário mínimo e ao aumento dos gastos públicos em saúde, educação e infraestrutura. Sobressaem-se, também, aquelas que visavam facilitar o acesso ao crédito às famílias e que concediam incentivos fiscais ao investimento privado. Tais políticas são apontadas como responsáveis pela redução da desigualdade de renda e pelo bom desempenho do mercado de trabalho (redução do desemprego), o que teria acelerado o crescimento do produto via expansão da demanda agregada, especialmente na primeira década dos anos 2000.

Não obstante, o processo de crescimento da economia brasileira desacelerou a partir de 2011, apresentando baixo crescimento em 2014 e fortes quedas no biênio 2015-16. Nesse período, houve uma piora geral dos indicadores econômicos, como o aumento do desemprego e da desigualdade e queda acentuada dos investimentos. Algumas explicações para tal cenário vão desde o desaquecimento do mercado externo, passando pelas mudanças no rumo das políticas macroeconômicas e pela condução do processo de redução da desigualdade até o contexto de crise política (Rugitsky, 2016). Em outras palavras, o debate se estende entre aqueles que, por um lado, sugerem que o ciclo de crescimento puxado sobretudo pelo consumo teria entrado em colapso, enquanto, por outro lado, muitos apontam que foram as mudanças de rumo da política macroeconômica que condicionaram forçosamente o fim desse ciclo.

Os modelos de crescimento neokaleckianos investigam justamente como a distribuição funcional da renda afeta a determinação da acumulação de capital e a demanda agregada (Onaran, Galanis, 2011; Lavoie, Stockhammer, 2013; Carvalho, Rezai, 2015; Lavoie, 1996, 2017). Com efeito, se prevalecer um regime de crescimento do tipo wage-led, então, políticas que aumentam a participação dos salários na renda, como a política de valorização real do salário mínimo, por exemplo, tendem a aumentar o grau de utilização da capacidade produtiva. Isso porque a demanda via consumo aumenta devido à diferença entre a propensão a consumir dos trabalhadores e dos capitalistas. Assim, a taxa de acumulação (crescimento do produto) se acelera.

No entanto, se prevalecer um regime de crescimento do tipo profit-led, em que a sensibilidade do investimento às variações da participação dos lucros na renda 
é elevada, e se a diferença entre as propensões a consumir entre capitalistas e trabalhadores for reduzida, um aumento da demanda via consumo induzida pelo aumento real do salário mínimo pode ser mais do que compensada pela queda dos investimentos, o que ocasiona uma redução do grau de utilização da capacidade produtiva. A justificativa é que a redução dos lucros das empresas tem efeitos negativos sobre o investimento por elas planejado (Bhaduri, Marglin, 1990).

Logo, a determinação do regime de crescimento que preponderou no período permite elucidar o debate acerca dos determinantes do crescimento econômico brasileiro e, ainda, avaliar as políticas macroeconômicas adotadas a partir dos seus efeitos esperados.

Diante do exposto, o objetivo do artigo é analisar o regime de crescimento econômico brasileiro que prevaleceu no período 2000-2015, levando em consideração a distribuição pessoal e funcional da renda. Em termos teóricos, foi utilizado o modelo neokaleckiano aumentado proposto por Palley (2016), pois ele permite uma análise mais abrangente dos efeitos da distribuição de renda, a exemplo do que aconteceu no Brasil. Para tanto, utilizamos indicadores de desigualdade pessoal da renda e dos rendimentos do trabalho. Como metodologia, utilizam-se vetores autorregressivos. A novidade está justamente na incorporação da dimensão pessoal da renda que ainda não foi considerada nos trabalhos que analisam o caso brasileiro (Gala; Araújo, 2012; Oreiro; Araújo, 2013; Feijó et al., 2015; Silva de Jesus, et al. 2017; Tomio, 2016; Gonçalves, 2017).

Para tanto, o artigo está dividido em seis seções, além desta introdução. A segunda seção contextualiza os regimes de crescimento e o desempenho da economia brasileira no período em análise. A seção seguinte apresenta o modelo teórico neokaleckiano proposto por Palley (2016). A quarta seção aborda a estratégia empírica, as variáveis selecionadas e a metodologia. Na quinta seção analisam-se os resultados e, por fim, apresentam-se as considerações finais.

\section{REGIMES DE CRESCIMENTO E A ECONOMIA BRASILEIRA ENTRE 2000 E 2015}

A teoria neo-kaleckiana analisa o papel da demanda efetiva em um contexto dinâmico o qual explicitamente considera o processo de distribuição da renda. Mais especificamente, a distribuição da renda torna-se um importante parâmetro para a determinação do nível de produto.

Os modelos básicos neokaleckianos foram inicialmente desenvolvidos por Rowthorn (1981) e Dutt $(1984,1997)$. O principal resultado dessa classe de modelos é o de que uma redução nos markups incorporados ao preço das firmas e, assim, no profit share, aumenta a taxa de utilização da capacidade, os lucros e a acumulação de capital. Em outras palavras, a demanda agregada, a acumulação de capital e o crescimento são wage-led. Bhaduri e Marglin (1990), entre outros, apresentaram pequenas variações dentro dessa classe de modelos alterando, por 
exemplo, os determinantes da função investimento. Com isso, foi possível derivar diferentes regimes potenciais de crescimento a depender da magnitude dos parâmetros.

Com a publicação do trabalho de Bhaduri e Marglin (1990) tem origem uma ampla agenda empírica. Os autores já tinham usado dados descritivos para um grupo de 16 economias capitalistas avançadas para ilustrar sua abordagem, no período entre 1960 e 1985, concluindo que houve alteração em termos do regime de crescimento durante a turbulenta década de 1970. Ou seja, as economias capitalistas avançadas saíram de um regime cooperativo com demanda e crescimento liderados pelos salários, em direção a um regime intermediário antagônico, com demanda liderada pelos salários, mas crescimento liderado pelos lucros.

Hein e Krämer (1997) estenderam a análise ao início dos anos 1990 para a França, Alemanha, Reino Unido e Estados Unidos, usando dados descritivos e calculando os valores cíclicos médios das principais variáveis. Os autores especulam sobre a existência de nova mudança no período, com o regime de crescimento nesses países passando a ser, novamente, liderados pelos salários durante o ciclo do início dos anos 1980 até início dos anos 1990. Para o Brasil, Araújo e Gala (2012) analisam o regime macroeconômico de crescimento no período 2002-2008. Os autores chegam à conclusão de que, quando se considera a demanda doméstica, o crescimento é wage-led. No entanto, quando se inclui a participação do setor externo, o crescimento brasileiro é profit-led, ou seja, ao se considerar investimento e exportações, a demanda agregada passa a responder de forma positiva ao aumento da participação dos lucros na renda.

Assim como para boa parte dessa literatura empírica, os resultados para o caso brasileiro são sensíveis ao período e aos métodos de análise utilizados (Oreiro, Araújo, 2013; Feijó et al., 2015; Silva de Jesus et al., 2017; Tomio, 2016; Gonçalves, 2017). Um ponto a ser ressaltado, no entanto, é que esses estudos não incorporam o papel da distribuição interpessoal da renda. Em outras palavras, a proposição básica é que, para obter resultados mais robustos, se faz necessária a inclusão, sob diferentes óticas, do conflito distributivo na análise da determinação do regime. Isso porque houve uma mudança na redistribuição de renda no período, principalmente do ponto de vista interpessoal. Por exemplo, os dados das pesquisas domiciliares utilizados para avaliar a distribuição funcional da renda mostram que no período entre 2000 e 2015 houve um aumento da participação dos salários na renda nacional, ao menos desde 2004ํ․ Nesse caso, a política de valorização do salário mínimo merece destaque como política (re)distributiva. O poder de compra dos assalariados aumentou no período em análise, com destaque para os anos de 2004 a 2010, em que a valorização real foi de 50,6\% (período 2000-2015). Ademais, foram implementadas uma série de políticas que visaram à redução da informalidade no mercado de trabalho, diminuindo o número de trabalhadores que recebiam menos de um salário mínimo.

\footnotetext{
${ }^{1}$ Vale lembrar que a análise da distribuição funcional da renda a partir dos dados fiscais mostrou que houve uma leve melhora entre 2000 e 2015 , com um aumento médio acumulado de 3,4\% da participação do fator trabalho na renda total, um aumento bem mais tímido do que se avaliava.
} 
Outro fator que contribuiu para essa mudança na estrutura distributiva foi a expansão dos programas de transferência de renda condicionada, tal qual o Bolsa Família, que, de acordo com Hoffman (2013), atende os estratos mais baixos da hierarquia da renda no país, com cerca de $90 \%$ dos benefícios destinados à população que compõe os $45 \%$ mais pobres. Já a expansão do acesso ao crédito também impulsionou o consumo (Schettini et al., 2012). No Brasil, o acesso ao crédito por pessoas físicas aumentou cerca de $90 \%$ em termos absolutos entre 2000 e 2010 (BCB, 2019). Santos et al. (2015) e Schettini et al. (2012) afirmam que essa expansão é decorrente do crescimento real dos rendimentos do trabalho, da redução da taxa de juros básica da economia e, também, do dinamismo no mercado imobiliário e das políticas habitacionais promovidas pelo governo.

A relação entre o aumento da renda disponível e a formalização no mercado de trabalho, particularmente quando atende as famílias com menor nível de renda, gera um círculo virtuoso. Os aumentos no salário mínimo e nas transferências públicas de renda geram demandas adicionais por serviços e bens manufaturados, propiciando novos empregos nestes setores e, por conseguinte, maior renda (Rugitsky, 2016). Esse parece ser o caso brasileiro. Os efeitos da redistribuição de renda no período são mais evidentes no mercado de trabalho. De acordo com o índice de Gini dos Rendimentos do Trabalho, houve redução da desigualdade em aproximadamente $12 \%{ }^{2}$.

Em resumo, é preciso levar em consideração o papel que mudanças na distribuição de renda exercem sobre os distintos componentes da demanda agregada e, por conseguinte, sobre o produto. Nesse contexto, o desafio é empreender uma análise que leve em consideração a relação entre distribuição de renda (interpessoal e funcional) e demanda agregada. A seção a seguir explicita essa relação na perspectiva dos modelos kaleckianos.

\section{EFEITOS DA DISTRIBUIÇÃO DE RENDA NOS REGIMES DE CRESCIMENTO}

Em decorrência da maior disponibilidade de dados sobre a distribuição de renda nos últimos anos, é crescente a literatura empírica que avalia a influência da desigualdade de renda no desempenho econômico. A maioria dos estudos empíricos recentes são para economias desenvolvidas (Onaran et al., 2011; Stockhammer et al., 2011; Stockhammer, Stehrer, 2011; Hein, Vogel, 2008; Naastepad, Storm, 2007; entre outros), com algumas exceções para países em desenvolvimento (Molero, 2011;

\footnotetext{
${ }^{2}$ Utilizando dados da distribuição pessoal da renda em uma análise para os EUA no período de 1967-2010, Carvalho e Rezai (2015) chegam à conclusão de que a propensão a poupar aumenta significativamente no quintil superior dos assalariados. Desse modo, uma redução da desigualdade de renda entre os trabalhadores resulta num regime de crescimento cada vez mais liderado pelos salários.
} 
Jetin, Kurt, 2011; Onaran, Stockhammer, 2005). Todos esses estudos utilizam dados sobre a distribuição funcional da renda.

O trabalho de Piketty (2014) evidencia a crescente desigualdade interpessoal de renda em diversos países, com uma apropriação da renda cada vez maior pelo topo da distribuição (pelos mais ricos). Por sua vez, Voitchovsky (2005) mostra que a desigualdade em diferentes partes da distribuição de renda tem efeitos distintos sobre o crescimento. Em outras palavras, o autor critica a utilização de uma estatística única de desigualdade para toda a distribuição, pois considera isso insuficiente para capturar os efeitos da desigualdade sobre o crescimento.

Palley $(2014,2016)$ argumenta que os modelos convencionais que incorporam o conflito "salário $v s$. lucro" ignoram o impacto de outras dimensões da distribuição de renda. O autor propõe um modelo alternativo neokaleckiano incorporando a desigualdade pessoal da renda, como apresentado a seguir.

O modelo convencional é descrito pelas seguintes equações:

$$
\begin{aligned}
& Y=W+\Pi \\
& W=[1-\mathrm{z}] \mathrm{Y} \\
& \Pi=\mathrm{z} Y \\
& \frac{I}{K}=I=\alpha_{0}+\alpha_{1} u+\alpha_{2} \mathrm{z}+\alpha_{3} \pi \quad \text { onde } \alpha^{\prime} \mathrm{s}>0 \\
& \pi=\mathrm{z} u \\
& \mathrm{z}=\mathrm{z}(\Psi), \quad z_{\Psi}>0 ; 0 \leq \mathrm{z} \leq 1 \\
& \frac{s}{K}=S=\beta_{K} \pi, \quad 0 \leq \beta_{K} \leq 1 \\
& \mathrm{I}=\mathrm{S} \\
& \mathrm{g}=\frac{I}{K}
\end{aligned}
$$

em que $\mathrm{Y}=$ renda nacional; $\mathrm{W}=$ massa salarial; $\Pi=$ lucros; $\mathrm{z}=$ parcela dos lucros na renda nacional; $\mathrm{I}=$ investimentos; $\mathrm{K}=$ estoque de capital; $\mathrm{S}=$ poupança dos capitalistas; $\mathrm{u}=$ taxa de utilização da capacidade $(\mathrm{Y} / \mathrm{K}) ; \pi=$ taxa de lucro $(\Pi / \mathrm{K})$; $\beta_{\mathrm{k}}=$ propensão a poupar dos capitalistas; $\psi=$ poder de mercado das firmas e outras variáveis que impactam positivamente a parcela dos lucros.

A equação (1) define que a renda nacional é dividida entre salários e lucros. As equações (2) e (3) determinam os salários e os lucros como uma parcela da renda nacional. O pressuposto é que todo o salário é pago aos trabalhadores e todo o lucro é pago aos capitalistas. A equação (4) determina a taxa de acumulação de capital como uma função positiva da utilização da capacidade, da participação dos lucros na renda e da taxa de lucro. A equação (5) determina que a taxa de lucro é igual ao produto da participação dos lucros e da taxa de utilização da capacidade. A equação (6) determina a participação dos lucros na renda como uma função positiva do poder de mercado das empresas que, por sua vez, influencia a determinação do 
preço. A equação (7) determina a taxa de poupança agregada, que depende apenas da poupança dos capitalistas, o que implica assumir a hipótese rígida de que os trabalhadores consomem todo o seu salário. A equação (8) é a condição de equilíbrio do mercado de bens e a equação (9) determina que a taxa de crescimento é igual à taxa de acumulação de capital.

Ao derivar a parcela do lucro (z) em relação ao grau de utilização da capacidade (u), obtêm-se a inclinação da curva IS:

$$
\frac{d z}{d u}=\frac{\left[S_{u}-I_{u}\right]}{S_{z}-I_{z}}=\frac{\beta_{K} \mathrm{z}-\alpha_{1}-\alpha_{3} \mathrm{z}}{\alpha_{2}+\left[\alpha_{3}-\beta_{K}\right] u}
$$

Em que $S_{u}=\beta_{K} z, S_{z}=\beta_{K} u, I_{u}=\alpha_{1}+\alpha_{3} z, I_{z}=\alpha_{2}+\alpha_{3} u$

Considerando que a condição de estabilidade do multiplicador keynesiano se mantém $\left(\left(\beta_{K}-\alpha 3>0\right)\right.$, então, $\left[S_{u}-I_{u}\right]>0$. Logo, a inclinação da IS depende exclusivamente do sinal do denominador, i.e., da sensibilidade relativa de investimento à participação dos lucros na renda. A IS é negativamente (positivamente) inclinada para uma economia wage-led (profit-led). A lógica é que um aumento na parcela de lucros reduz (aumenta) a demanda agregada $(\mathrm{I}<\mathrm{S})$, causando redução (expansão) na utilização da capacidade instalada (Palley, 2016).

Tabela 1: Condições descrevendo regimes de demanda profit-led, wage-led e regimes conflitantes

\begin{tabular}{cccc}
\hline & $\begin{array}{c}\text { Utilização da } \\
\text { capacidade }\end{array}$ & $\begin{array}{c}\text { Resposta } \\
\text { Investimento-Poupança }\end{array}$ & $\begin{array}{c}\text { Taxa } \\
\text { de crescimento }\end{array}$ \\
\hline Profit-led & $\mathrm{u}_{\mathrm{z}}>0$ & $\mathrm{I}_{\mathrm{z}}-\mathrm{S}_{\mathrm{z}}>0$ & $\mathrm{I}_{\mathrm{u}} \mathrm{u}_{\mathrm{z}}+\mathrm{I}_{\mathrm{z}}>0$ \\
Wage-led & $\mathrm{u}_{\mathrm{z}}<0$ & $\mathrm{I}_{\mathrm{z}}-\mathrm{S}_{\mathrm{z}}<0$ & $\mathrm{I}_{\mathrm{u}} \mathrm{u}_{\mathrm{z}}+\mathrm{I}_{\mathrm{z}}<0$ \\
Conflitante & $\mathrm{u}_{\mathrm{z}}<0$ & $\mathrm{I}_{\mathrm{z}}-\mathrm{S}_{\mathrm{z}}<0$ & $\mathrm{I}_{\mathrm{u}} \mathrm{u}_{\mathrm{z}}+\mathrm{I}_{\mathrm{z}}>0$ \\
\hline
\end{tabular}

Fonte: Elaboração própria a partir de Palley (2016).

Mudanças na participação dos lucros tornam o numerador maior, aumentando o valor absoluto da inclinação da IS. Nesse caso, se a economia for wage-led, um aumento na participação dos lucros pode potencialmente deslocar a economia de um regime liderado pelos salários para um regime conflitante. Por outro lado, se a economia for profit-led, uma participação cada vez maior dos lucros na renda nacional torna a economia marginalmente menos "lucrativa", porque a curva IS torna-se cada vez mais inclinada. Embora a função de investimento seja linear em $\mathrm{z}$, a inclinação da IS é não linear, consequentemente, além do sinal da mudança na participação dos lucros, o tamanho da participação dos lucros também é importante na capacidade de resposta da economia.

$\mathrm{Na}$ versão entendida do modelo, Palley (2016) incorpora o fato de que tanto trabalhadores como capitalistas recebem lucros e salários, o que afeta a poupança agregada, mas não a função investimento. Reescrevendo a função da poupança, tem-se: 


$$
\begin{aligned}
& \varphi_{K}+\varphi_{L}=1 \\
& \varphi_{K}+\varphi_{L}=1, \sigma_{K}>\varphi_{K} ; \sigma_{L}>\varphi_{L} ; \sigma_{K}>\sigma_{L} ; \varphi_{K}<\varphi_{L} \\
& S=S_{K}+S_{L} \\
& S_{K}=\beta_{K}\left\{\left[1-\varphi_{L}\right][1-z] u+\left[1-\sigma_{L}\right] z u\right\}, 0<\beta_{L}<\beta_{K} \leq 1 \\
& S_{L}=\beta_{L}\left\{\varphi_{L}[1-\mathrm{z}] u+\sigma_{L} \mathrm{z}, \quad 0<\beta_{L} \leq 1\right.
\end{aligned}
$$

em que $\varphi_{\mathrm{K}}=$ participação dos gestores capitalistas na massa salarial; $\varphi_{\mathrm{L}}=$ participação dos trabalhadores na massa salarial; $\sigma_{\mathrm{K}}=$ participação nos lucros dos capitalistas-gerentes, $\sigma_{\mathrm{L}}=$ participação nos lucros dos trabalhadores, $S_{\mathrm{K}}=$ taxa de poupança dos capitalistas-gerentes; $S_{\mathrm{L}}=$ taxa de poupança dos trabalhadores; $\beta_{\mathrm{K}}=$ propensão a poupar dos gestores capitalistas; e $\beta_{\mathrm{L}}=$ propensão a poupar dos trabalhadores. As restrições sobre o tamanho das participações acionárias tanto dos assalariados como dos capitalistas-gerentes repousam na hipótese de que os gerentes capitalistas detêm parte do estoque de capital e obtêm a maior parte de sua renda advinda dos lucros, enquanto os trabalhadores obtêm a maior parte de sua renda advinda dos salários.

Combinando as equações (12), (13) e (14), pode-se reescrever a função da poupança agregada como segue:

$$
S=\beta_{L}\left\{\varphi_{L}[1-\mathrm{z}] u+\sigma_{L} \mathrm{z} u\right\}+\beta_{K}\left\{\left[1-\varphi_{L}\right][1-\mathrm{z}] u+\left[1-\varphi_{L}\right] \mathrm{z} u\right\}
$$

Diferenciando (15) com relação a $z, \varphi_{\mathrm{L}}$ e $\sigma_{\mathrm{L}}$, tem-se:

$$
\begin{aligned}
& \frac{d s}{d \mathrm{z}}=\left[\beta_{L}-\beta_{K}\right]\left[\sigma_{L}-\sigma_{K}\right] u>0 \\
& \frac{d s}{d \sigma_{L}}=\left[\beta_{L}-\beta_{K}\right][1-\mathrm{z}] u<0 \\
& \frac{d s}{d \sigma_{L}}=\left[\beta_{L}-\beta_{K}\right] \mathrm{z} u<0
\end{aligned}
$$

Ou seja, um aumento na participação dos lucros na renda total aumenta a poupança agregada. A lógica é que há uma transferência dos salários para os lucros. Os trabalhadores recebem uma parcela menor dos lucros do que a referente a salários $(\sigma \mathrm{L}<\phi \mathrm{L})$, o que efetivamente transfere renda dos trabalhadores para os capitalistas, aumentando a propensão a poupar. Um aumento na participação dos trabalhadores na massa salarial transfere renda dos gerentes-capitalistas, que poupam mais, para os trabalhadores que possuem uma menor propensão a poupar, diminuindo a poupança agregada.

A Tabela 2 sintetiza os efeitos macroeconômicos das mudanças nos parâmetros distributivos no modelo aumentado. 
Tabela 2: Efeitos da distribuição da renda na utilização da capacidade e na taxa de crescimento da economia

\begin{tabular}{cccc}
\hline & Wage-Led & Conflitante & Profit-Led \\
\hline$d u / d z$ & - & - & + \\
$d g / d z$ & - & + & + \\
$d u / d \varphi L$ & + & + & + \\
$d g / d \varphi L$ & + & + & + \\
$d u / d \sigma L$ & + & + & + \\
$d g / d \sigma L$ & + & + & + \\
\hline
\end{tabular}

Fonte: Elaboração própria a partir de Palley (2016).

Qualquer que seja o regime de crescimento da economia, a redistribuição dos salários dos gerentes-capitalistas para os trabalhadores tem um impacto positivo na taxa de crescimento, porque por mais que haja uma redução da poupança agregada, em contrapartida há um aumento da demanda agregada.

A partir da Tabela 2 pode-se inferir que os efeitos da mudança na participação dos lucros dependem do regime de crescimento. Desse modo, depende também da inclinação e do escalonamento da curva IS. A inclinação da IS é dada por:

$$
\frac{d z}{d u}=\frac{\left[S_{u}-I_{u}\right]}{S_{z}-I_{z}}=\frac{\left\{\beta_{L}\left\{\varphi_{L}[1-\mathrm{z}]+\varphi_{L} z\right\}+\beta_{K}\left\{\left[1-\varphi_{L}\right][1-z]+\left[1-\sigma_{L}\right] \mathrm{z}\right\}-\alpha_{1}-\alpha_{3} z\right.}{\left\{\alpha_{2}+\left\{\alpha_{3}-\beta_{L}\left\{\sigma_{L}-\varphi_{L}\right\}-\beta_{K}\left\{\left[1-\sigma_{L}\right]-\left[1-\varphi_{L}\right]\right\}\right\} u\right\}}
$$

A inclinação da IS depende dos três parâmetros de distribuição $(z, \varphi \mathrm{L}$ e $\sigma \mathrm{L})$. Como antes, se a condição de estabilidade do multiplicador keynesiano for válida, o numerador é positivo e o sinal da inclinação depende exclusivamente do sinal do denominador. A economia é wage-led ou conflitante se o denominador for negativo e profit-led se for positivo. Os aumentos na participação nos lucros $(z)$ não têm efeito sobre o denominador. O aumento da participação dos trabalhadores nos lu$\operatorname{cros}\left(\sigma_{\mathrm{L}}\right)$ aumenta o denominador, aumentando a possibilidade de que ele se torne positivo e que a economia seja profit-led. Isso pode parecer contraditório, mas a lógica é que se os trabalhadores ganham uma parcela maior dos lucros, dada sua menor propensão a poupar, uma parcela maior dos lucros é consumida, dando um maior poder aos lucros de impulso à demanda agregada. Já o aumento dos trabalhadores na massa salarial $\left(\phi_{\mathrm{L}}\right)$ reduz o denominador, tornando mais provável que seja negativo e a economia seja wage-led.

Como as mudanças na distribuição dos salários podem mudar o regime de crescimento de uma economia, mudanças na distribuição interpessoal de renda também podem ter este efeito. A desigualdade interpessoal da renda é definida pela razão entre a renda total do gestor-capitalista e a renda total do trabalhador, como segue:

$$
\Omega=\mathrm{Y}_{\mathrm{K}} / \mathrm{Y}_{\mathrm{L}}
$$


O rendimento total dos gestores-capitalistas e dos trabalhadores é definido como:

$$
\begin{aligned}
& Y_{K}=\varphi_{K}[1-z] Y+\sigma_{K} z Y \\
& Y_{L}=\varphi_{L}[1-z] Y+\sigma_{L} z Y \\
& Y=Y_{K}+Y_{L}
\end{aligned}
$$

Substituindo as equações (17) e (18) em (16), tem-se:

$$
\Omega=\frac{\left\{\varphi_{K}[1-z]+\sigma_{K} z\right\}}{\left\{\varphi_{Z}[1-z]+\sigma_{L} z\right\}}=\Omega\left(z, \varphi_{K}, \varphi_{L}\right)
$$

Pode-se, então, mostrar que um aumento da participação dos gestores-capitalistas na massa salarial aumenta a desigualdade $\left(\mathrm{d} \Omega / \mathrm{d} \varphi_{\mathrm{K}}>0\right)$, assim como um aumento da participação destes nos lucros $\left(\mathrm{d} \Omega / \mathrm{d} \sigma_{\mathrm{K}}>0\right)$. O corolário é que um aumento da participação dos trabalhadores na massa salarial diminui a desigualdade $(\mathrm{d} \Omega /$ $\mathrm{d} \varphi_{\mathrm{L}}<0$ ), do mesmo modo que um aumento da participação dos trabalhadores nos lucros $(\mathrm{d} \Omega / \mathrm{d} \sigma \mathrm{L}<0)$.

\section{REGIME DE CRESCIMENTO PARA A ECONOMIA BRASILEIRA NO PERÍODO 2000-2015}

A estimativa do modelo que busca captar o regime de crescimento para o Brasil no período de 2001 a 2015 será realizada a partir das seguintes funções:

$$
\begin{aligned}
& g=f(u ; \pi ; \mathrm{z}) \\
& S=f(\Pi ; W) \\
& \Omega=f\left(\mathrm{z}, \varphi_{K}, \varphi_{L}\right)
\end{aligned}
$$

em que $g$ é a taxa de crescimento do investimento, como função da utilização da capacidade instalada $(u)$, da taxa de lucro $(\pi)$ e da participação dos lucros na renda $(z)$. A função da poupança $(S)$ determinada pela poupança dos trabalhadores (W como a participação dos salários na renda) e dos capitalistas ( $\Pi$ como a participação dos lucros na renda). A desigualdade pessoal da renda $\Omega$ (medida pelo índice de Gini Renda) como função da participação dos trabalhadores na massa salarial $\left(\varphi_{\mathrm{L}}\right)$, da participação dos capitalistas na massa salarial $\left(\varphi_{\mathrm{k}}\right)$ e da participação dos lucros na renda (z). A equação reduzida foi utilizada pela indisponibilidade de dados referentes à renda do capital.

Ressalta-se que, em termos empíricos, a literatura divide-se entre dois métodos: i) a estimação de equações únicas (Bowles, Boyer, 1995); e ii) a dos modelos de vetores autorregressivos (Stockhammer, Onaran, 2004; Onaram, Stockhammer, 2005). Este trabalho seguirá a segunda abordagem, tendo em vista a natureza dos dados disponíveis e o objetivo do trabalho, que inclui estabelecer relações lineares entre as variáveis e suas defasagens. A Tabela 3 abaixo sintetiza a escolha das variáveis utilizadas. 


\begin{tabular}{|c|c|c|}
\hline Variável & Descrição & Fonte \\
\hline Estoque de Capital (K) & $\begin{array}{l}\text { Estoque de capital fixo produtivo. Corresponde à soma do } \\
\text { estoque em máquinas e equipamentos mais o estoque em } \\
\text { construções não residenciais }{ }^{3} \text {. }\end{array}$ & Ipeadata \\
\hline Taxa de Lucro $(\pi)$ & $\begin{array}{l}\text { Corresponde à massa de lucro bruto macroeconômico } \\
\text { dividida pelo estoque de capital fixo produtivo total (K) da } \\
\text { economia brasileira. A massa de lucro bruto foi calculada pela } \\
\text { diferença entre o PIB a custo de fatores e a massa salarial. } \\
\text { Esta última incluiu a remuneração dos empregados e dos } \\
\text { trabalhadores por conta própria e as contribuições sociais }{ }^{4} \text {. }\end{array}$ & IBGE e Ipeadata \\
\hline $\begin{array}{l}\text { Taxa de acumulação de } \\
\text { capital fixo produtivo (g) }\end{array}$ & $\begin{array}{l}\text { Corresponde à razão entre a formação bruta de capital fixo e } \\
\text { o estoque de capital fixo produtivo }(\mathrm{K}) \text { disponível à economia } \\
\text { brasileira. }\end{array}$ & IBGE e Ipeadata \\
\hline $\begin{array}{l}\text { Nível de utilização da } \\
\text { capacidade produtiva } \\
\text { instalada (u) }\end{array}$ & $\begin{array}{l}\text { Nuci. Corresponde às séries dessazonalizadas produzidas } \\
\text { pela Confederação Nacional da Indústria (CNI). }\end{array}$ & Ipeadata \\
\hline Poupança (S) & $\begin{array}{l}\text { Corresponde à poupança total da economia, que aqui será } \\
\text { utilizada em escala logarítimica. }\end{array}$ & Ipeadata \\
\hline Investimento (I) & Corresponde à formação bruta de capital fixo. & Ipeadata \\
\hline Total se Salários (W) & $\begin{array}{l}\text { Corresponde ao somatório dos Rendimentos do Trabalho } \\
\text { na Renda Nacional em termos percentuais. }\end{array}$ & $\begin{array}{l}\text { World Wealth and } \\
\text { Income Database }\end{array}$ \\
\hline Total de Lucros (L) & $\begin{array}{l}\text { Corresponde ao somatório dos Lucros na Renda Nacional em } \\
\text { termos percentuais. }\end{array}$ & $\begin{array}{l}\text { World Wealth and } \\
\text { Income Database }\end{array}$ \\
\hline Profit Share (z) & Corresponde à participação dos Lucros na Renda Nacional. & $\begin{array}{l}\text { World Wealth and } \\
\text { Income Database }\end{array}$ \\
\hline $\begin{array}{l}\text { Desigualdade Pessoal } \\
\text { da Renda }(\Omega)\end{array}$ & $\begin{array}{l}\text { Corresponde ao Índice de Gini da Renda calculado pela } \\
\text { metodologia DINA6. }\end{array}$ & Várias fontes \\
\hline
\end{tabular}

${ }^{3}$ A partir de 2008 os valores foram estimados com base na criação de estoque de capital e depreciação.

${ }^{4} \mathrm{O}$ cálculo da taxa de lucro se baseou no trabalho de Araújo e Gala (2012).

${ }^{5}$ A base de dados World Wealth and Income Database foi escolhida por trazer uma série harmonizada de acordo com a metodologia Distributional National Accounts (DINA) para o cálculo dos percentis de renda na construção do índice de Gini, e tem plena correspondência com as Contas Nacionais brasileiras.

${ }^{6}$ Os conceitos e métodos utilizados pelo WID seguem a estrutura do Distributional National Accounts (DINA) e são definidos usando os mesmos conceitos que os propostos nas últimas diretrizes internacionais sobre as contas nacionais macroeconômicas. Conforme estabelecido pelo Sistema de Contas Nacionais da ONU (SNA) de 2008 (ver site de Contas Nacionais da ONU e página de diretrizes on-line SNA 2008 e SNA 2008 pdf guideline). Utilizam códigos de classificação do SNA 2008 ou do Sistema Europeu de Contas (ESA 2010). Para alguns países, e/ou para períodos mais antigos, segue ainda o antigo sistema de diretrizes internacionais, nomeadamente SNA 1993 (ou a versão europeia, SEC 1995) (Alvaredo, Atkinson, Chancel, Piketty, Saez e Zucman, 2017). 


\begin{tabular}{c|c|c}
\hline $\begin{array}{c}\text { Participação dos } \\
\text { capitalistas nos salários } \\
\left(\varphi_{k}\right)\end{array}$ & $\begin{array}{r}\text { Será utilizada uma proxy na qual corresponde à participação } \\
\text { dos gerentes capitalistas na massa salarial. }\end{array}$ & DIRPF \\
\hline $\begin{array}{c}\text { Participação dos } \\
\text { trabalhadores na massa } \\
\text { salarial }\left(\varphi_{L}\right)\end{array}$ & $\begin{array}{r}\text { Será utilizada uma proxy na qual corresponde à participação } \\
\text { dos demais trabalhadores na massa salarial. }\end{array}$ & DIRPF \\
\hline
\end{tabular}

Fonte: Elaboração Própria.

Para estimar o modelo VAR, primeiramente, realizou-se o teste de raiz unitária, a fim de verificar se as variáveis seguem uma trajetória de processo estocástico estacionário. Para tanto, foi utilizado o teste de raiz unitária Dickey-Fuller Aumentado (ADF), em nível, cuja hipótese nula (H0) é de que a série possui raiz unitária. ou seja, é não estacionária. Os resultados mostram que todas as variáveis não são estacionárias, ou seja, não rejeitam a hipótese nula de que possuem raiz unitária ${ }^{7}$ Para corrigir esse problema, tiramos a primeira diferença das variáveis e realizamos novamente o teste de raiz unitária. Após a análise de estacionaridade das variáveis, testamos o número ótimo de lags para cada função a ser estimada, a fim de verificar quantas defasagens ajustam melhor o modelo. Para tanto, realizamos os seguintes testes: Lag Order Selection Criteria e Lag Exclusion Wald Tests. Como não houve inconsistência nos resultados dos dois testes, a escolha das defasagens que melhor ajustam os modelos foram: 1 lag para as funções $g$ e $\Omega$ e 4 lags para a função da poupança $(S)$.

Para avaliar a normalidade dos resíduos, realizamos o diagnóstico para autocorrelação residual por meio do Residual Serial Correlation LM Tests. A hipótese nula dos testes é de ausência de autocorrelação até o lag especificado. Assim, os resultados indicaram que não se rejeita a hipótese nula de que não há autocorrelação nos resíduos das variáveis em regressão.

Após a realização desses testes ${ }^{8}$, é possível avaliar quais modelos apresentam melhor especificação e quais geram resultados mais confiáveis e robustos. Isso é realizado a seguir a partir das análises das funções “impulso-resposta”.

\section{ANÁLISE DOS RESULTADOS}

A taxa de acumulação de capital $(g)$ responde positivamente a um impulso em si mesma e, em menor escala, na taxa de utilização da capacidade instalada (Figura 1). Ou seja, como esperado, é possível argumentar que em termos de produção a economia

\footnotetext{
${ }^{7}$ Com exceção da capacidade utilizada, que se mostrou estacionária para um nível de 5\% de confiança.

${ }^{8} \mathrm{O}$ teste de cointegração não foi especificado, pois, de acordo com a literatura vigente, esse teste se faz necessário para séries mais longas, que não é o caso dos dados deste trabalho.
} 
brasileira entre 2001 e 2015 respondeu positivamente ao crescimento da acumulação de capital e ao aumento no grau utilização da capacidade produtiva instalada.

Figura 1: Resposta da taxa de acumulação de capital a um choque na utilização da capacidade, na taxa de lucro e na participação dos lucros na renda

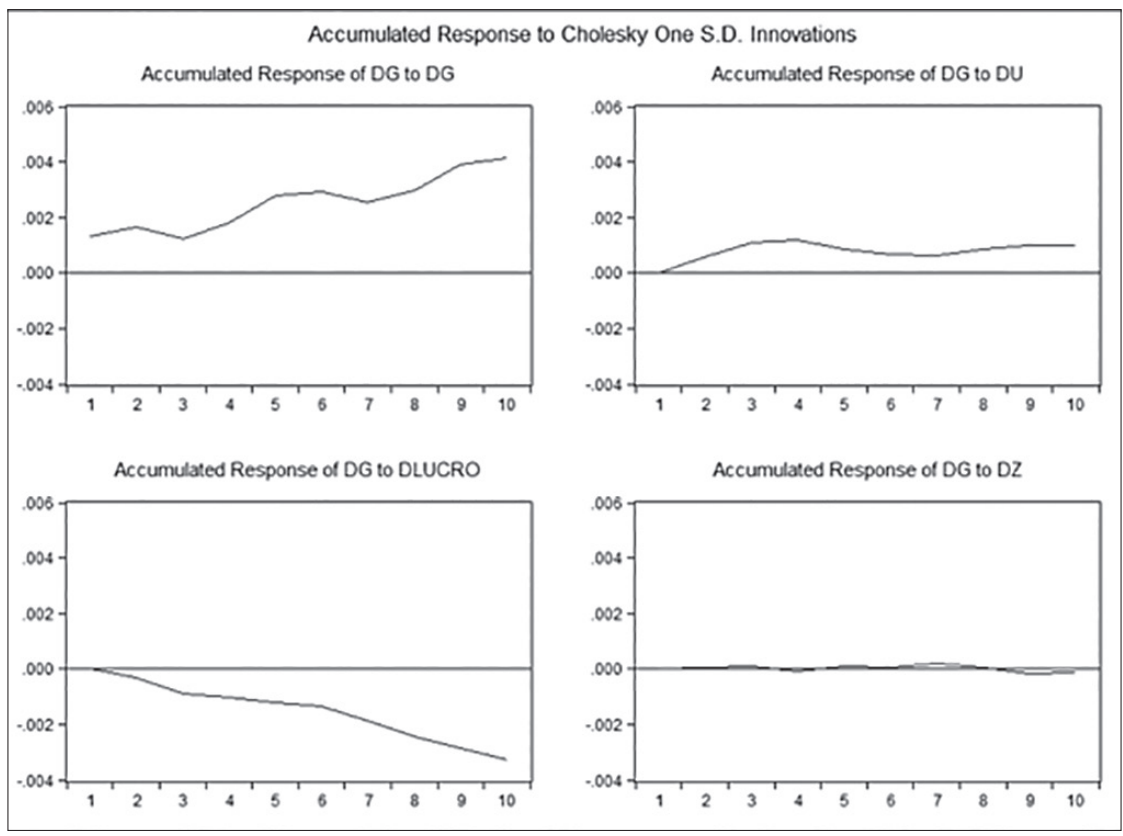

Fonte: Elaboração própria a partir do Eviews.

A resposta de $g$ à taxa de lucro foi negativa e o aumento da participação dos lucros na renda pouco ou nada influencia na acumulação de capital. Uma possível explicação para tal cenário é que no país um aumento nos lucros favorece a poupança e não o investimento produtivo.

A Figura 2 mostra a resposta da poupança $(S)$ a choques na participação dos lucros e dos salários na renda. Assume-se que os trabalhadores têm propensão marginal a poupar menor do que os capitalistas. Portanto, espera-se que um aumento da participação dos salários tenha um impacto negativo na poupança, bem como um aumento na participação dos lucros tenha o efeito oposto, ou seja, um aumento na poupança agregada. $\mathrm{O}$ caso brasileiro confirma essa hipótese. A poupança responde positivamente a um aumento na participação dos lucros. Em um primeiro momento, essa variação se mostra mais acentuada, com impactos marginais menores com o decorrer do tempo.

O aumento da participação dos salários na renda apresenta relação negativa com a poupança. Um choque inicial pró-salário tem um impacto negativo mais abrupto na poupança. Assim, pode-se inferir que, em princípio, um aumento na renda dos trabalhadores impulsiona mais aceleradamente o consumo. 
Figura 2: Resposta da poupança a um choque

na participação dos lucros e dos salários na renda

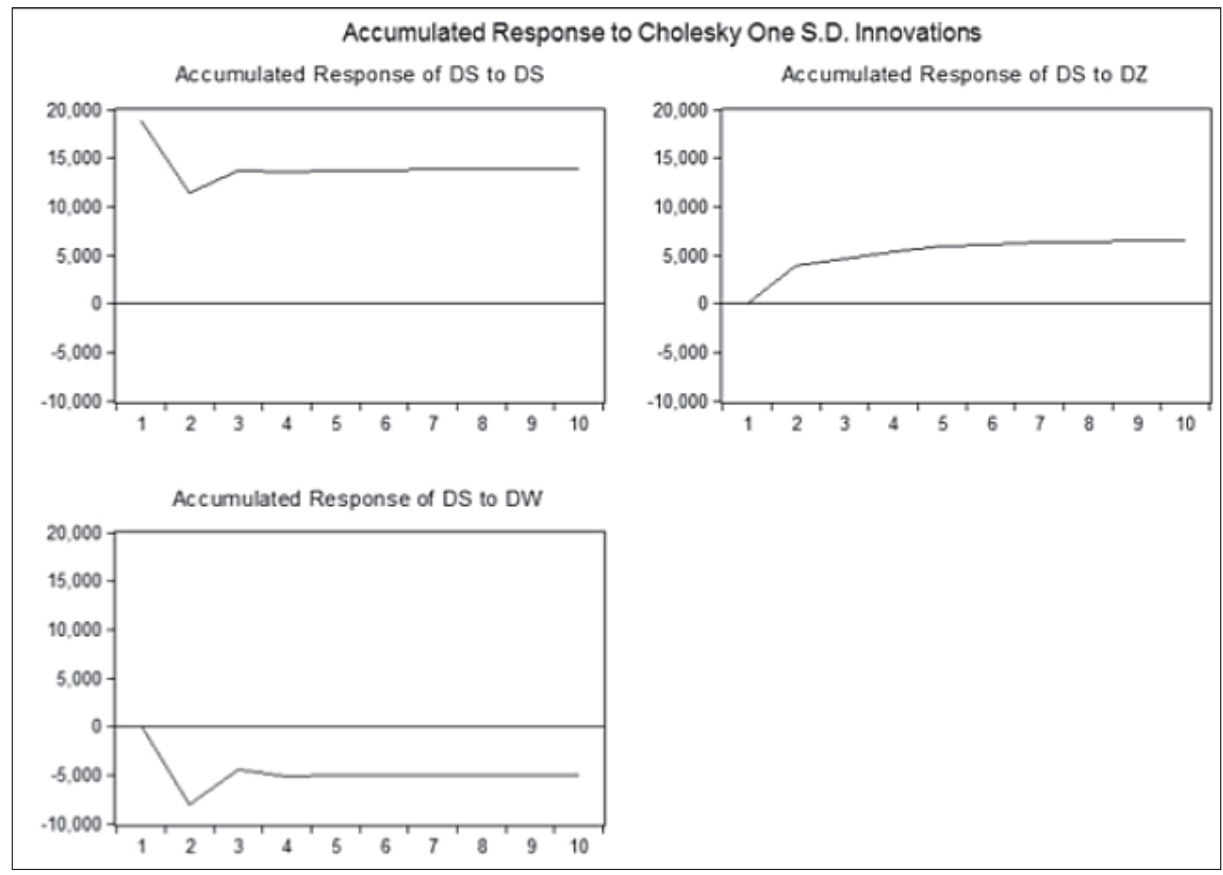

Fonte: Elaboração própria a partir do Eviews.

A seguir, serão considerados os efeitos sobre a distribuição interpessoal da renda $(\Omega)$, medidos aqui pelo índice de Gini Renda. Também serão analisados os efeitos de mudanças na participação dos lucros na renda $(z)$, da participação dos gerentes capitalistas nos salários $\left(\varphi_{K}\right.$, no gráfico $\left.\mathrm{f}_{-} \mathrm{K}\right)$, e dos trabalhadores na massa salarial $\left(\varphi_{L}\right.$, no gráfico f_L) (Figura 3 ).

Os resultados mostram o caráter inercial da desigualdade de renda. Isso pode ser explicado pelo fato de que, quanto maior a concentração de renda de uma sociedade, mais ela tende a se perpetuar, haja vista que os ganhos de capital dos lucros e da riqueza são maiores que os ganhos dos salários (Piketty, 2014). No caso do Brasil, que é um dos países com maiores indicadores de desigualdade do mundo (Souza, 2017; Morgan, 2018), essa lógica se confirma. Essa mesma tendência também é verificada ante um choque na participação dos lucros $(z)$ na renda total. O índice de desigualdade aumenta, com intensidade em um período mais curto de tempo, quando do aumento de $z$. 
Figura 3: Resposta do índice de Gini Renda a um choque na

participação dos lucros na renda, da participação dos

gerentes capitalistas e dos trabalhadores na massa salarial

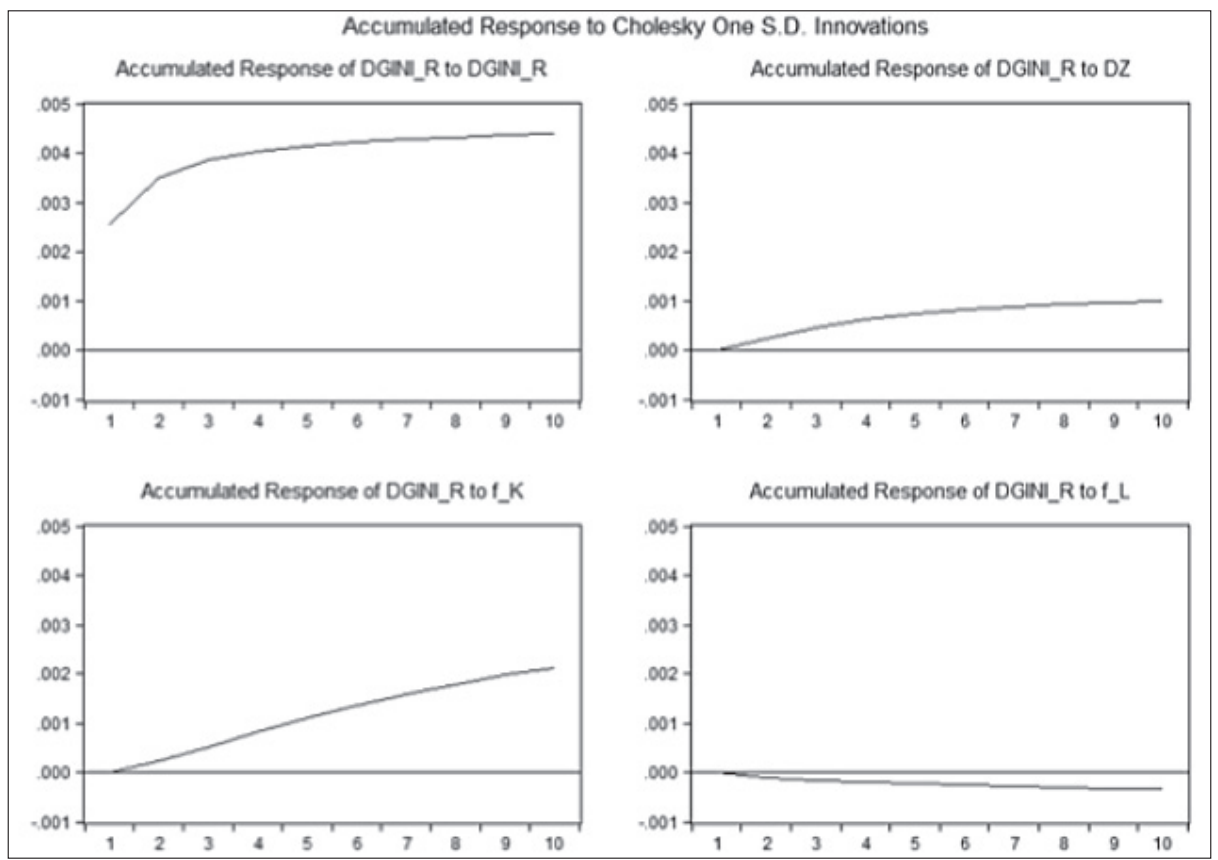

Elaboração própria a partir do Eviews.

Um choque na participação dos gerentes capitalistas na massa salarial tem relação positiva e crescente com a desigualdade total da renda. A presença desse grupo de trabalhadores, que recebem uma renda mista composta por salários e lucros e que compõem a categoria dos "super salários", favorece a concentração da renda.

A resposta do índice de desigualdade da renda ao impulso do aumento da participação dos trabalhadores na massa salarial é negativa, ou seja, $\Omega$ se reduz diante da ampliação dos trabalhadores "comuns" na apropriação total dos salários na economia. O efeito sobre $\Omega$ é mais evidente em um prazo mais longo de tempo, a julgar por considerarmos apenas a participação destes na massa salarial e não uma ampliação dos salários na renda total. Avalia-se que o impacto total da transferência da renda dos "gerentes-capitalistas" para os trabalhadores "comuns" perde efeito ante a composição do mercado de trabalho brasileiro, cuja grande maioria $(88 \%)^{9}$ é composta por trabalhadores "comuns".

\footnotetext{
${ }^{9}$ Esse valor teve por base os dados da Declaração Anual do Imposto de Renda Pessoa Física (DIRPF), que pode estar subestimado, pois cobrem aproximadamente $45 \%$ da renda nacional e abrangem cerca de $30 \%$ do total de trabalhadores do país, e tende a subestimar os trabalhadores e seus rendimentos na base da distribuição bem como a informalidade no mercado de trabalho, ou seja, é pouco representativa dos $50 \%$ mais pobres.
} 
A seguir, apresenta-se uma síntese dos resultados dos efeitos teóricos esperados em cada regime de crescimento que vão nos auxiliar a elaborar um diagnóstico para o Brasil no período em análise (Tabela 4).

Tabela 4: Diagnóstico do Regime de Crescimento para o Brasil (2001-2015)

\begin{tabular}{lcccc}
\hline & Wage-Led & Conflitante & Profit-Led & Brasil \\
\hline$d u / d z$ & - & - & + & Inconclusivo \\
$d g / d z$ & - & + & + & Inconclusivo \\
$d u / d \Omega$ & - & - & - & - \\
$d g / d \Omega$ & - & - & - & - \\
$d S / d \Omega$ & + & + & + & + \\
$d u / d z-d S / d z$ & - & - & + & - \\
$d g / d z-d S / d z$ & - & - & + & - \\
\hline
\end{tabular}

Fonte: Elaboração própria.

As variações na utilização da capacidade instalada $(u)$ e na taxa de acumulação de capital $(g)$ mostraram movimentos bastantes semelhantes dada uma variação na participação dos lucros na renda nacional $(z)$. No curto prazo, a resposta de $g$ e $u$ a um impulso em $z$ apresentou-se nula e, em períodos mais longos, alternou em pequenos movimentos de respostas positiva e negativa. Logo, esse conjunto de parâmetros para o Brasil é inconclusivo.

O modelo neokaleckiano assume que um aumento da desigualdade total da renda diminui a demanda agregada, que, por sua vez, tem impactos negativos em $u$ e $g$, ao passo que há um aumento da poupança agregada. A hipótese é que quanto maior o grau de desigualdade total da renda $(\Omega)$, menor a participação dos trabalhadores “comuns" na massa salarial, cuja propensão a consumir é maior, portanto, menor a demanda agregada. Desse modo, o aumento de $\Omega$ deve impactar negativamente a taxa de acumulação de capital $(g)$ e a taxa de utilização da capacidade instalada $(u)$, porém aumenta a poupança agregada. Os dados mostram que esse cenário se verifica para o Brasil.

Em síntese, os resultados são inconclusivos. O resultado sobre a poupança dá alguma indicação adicional de que o regime de crescimento da economia brasileira no período pode ser caracterizado como wage-led ou conflitante.

\section{O MODELO BRASILEIRO À LUZ DOS RESULTADOS ENCONTRADOS}

A reversão do ciclo de crescimento após 2015 põe em dúvida qual seria o regime de crescimento da economia brasileira. Nesse caso, a discussão se estende a quais políticas econômicas poderiam ter sido adotadas para se evitar o aprofundamento da crise. Existe, portanto, um profícuo debate na literatura que vamos resumir con- 
siderando a perspectiva de dois modelos: i) o modelo export leg growth, que admite que a evolução das exportações é a principal componente da demanda final e, por conseguinte, o principal condutor do crescimento econômico, por permitir ganhos de produtividade e incorporação de progresso técnico, ao mesmo tempo em que induz a expansão sustentável da demanda de forma compatível com o equilíbrio intertemporal da conta-corrente (restrição externa) do Balanço de Pagamentos (Thirwall, 1979; Mccombie, Thirwall, 1994; Bresser-Pereira, Gala, 2012; OreirO et al., 2012); e ii) o modelo de crescimento wage-led, em que políticas de distribuição da renda em prol dos salários criam as condições para o crescimento de longo prazo (Freitas, Dweck, 2013; Serrano, Suma, 2012, 2015).

Por conseguinte, considerando os resultados acima e o debate nacional, entende-se que é possível avançar nessa discussão, em primeiro lugar, reconhecendo as limitações das próprias abordagens; e, em segundo, avançando na discussão acerca da viabilidade de construção de um modelo econômico baseado no crescimento liderado pelos salários e pelas exportações, como defendido por Ferrari Filho e Fonseca (2015). O ponto de partida é pensar a economia brasileira em um contexto de uma economia aberta. Assim, ao mesmo tempo em que o comportamento das exportações não pode explicar a crise vivenciada no final do período analisado, não se pode ignorar a sua capacidade de dinamizar o produto e, mais, amenizar o conflito distributivo ao permitir a manutenção - ou impedir uma queda mais acentuada - dos lucros na economia.

Assim, uma saída perpassa por aumentar os ganhos no comércio internacional ${ }^{10}$. Ou seja, é preciso crescer economicamente ganhando espaço em termos do volume e da complexidade das exportações, a fim de que se possa aumentar a participação nacional nos lucros internacionais. Ademais, em um contexto de baixo crescimento econômico, políticas voltadas a promover a redistribuição funcional da renda são limitadas como instrumento capaz de induzir a aceleração da expansão do produto. Esse limite torna-se ainda mais rígido quando tal política combina, como no caso brasileiro, valorização do salário mínimo e valorização da taxa real de câmbio, as quais, conjuntamente, acabam por tencionar a redução dos lucros a partir do aumento do custo unitário do trabalho e da impossibilidade de acesso ao mercado internacional. Além doisso, impõe limites à própria política de valorização sustentada dos salários ao longo do tempo, pois induz uma mudança estrutural regressiva que restringe a capacidade de produção e a própria formalização do mercado de trabalho. Nesse caso, destaca-se a interconexão entre a política cambial e as políticas de redistribuição de renda, de modo que a manutenção de uma taxa real de câmbio estável e competitiva ao longo do tempo é fundamental.

Evidentemente, existem outras questões apontadas pela literatura relacionadas aos possíveis efeitos inflacionários (pass through) e/ou perdas que segmentos produtivos que utilizam insumos importados podem incorrer a partir da implementação de uma

\footnotetext{
10 Obviamente, há diferentes outras formas de alcançar este objetivo, como as mudanças de cunho institucional, por exemplo.
} 
política de manutenção do câmbio real competitivo. Contudo, em primeiro lugar, os dados mostram que o efeito pass through é pequeno para a economia brasileira. Em segundo, é preciso considerar que, ainda que uma desvalorização real do câmbio possa frear no curto prazo o aumento do salário real, o aumento da produção do setor tradeable tende a aumentar a produtividade média da economia e acelerar a criação de empregos formais os quais, por sua vez, permitem - pressionam - a elevação dos salários no médio prazo ${ }^{11}$.

O conflito distributivo tem uma dimensão setorial e responde a mudanças na estrutura produtiva ${ }^{12}$. Então, mesmo considerando que o processo de crescimento seja liderado pela demanda (demand-led), é preciso ter em conta políticas capazes de induzir mudanças estruturais pró-crescimento. Esse é o caso da já mencionada política cambial, mas também é das políticas de investimento e dos gastos governamentais.

É nesse contexto que as políticas de contração fiscal, com a redução geral dos gastos públicos, devem ser avaliadas no período. Ao mesmo tempo em que essas políticas levaram à redução da demanda agregada e afetaram negativamente as expectativas do setor empresarial, elas puseram em xeque a continuidade das políticas de redistribuição da renda em prol dos salários. Por um lado, porque o desemprego começou a se elevar. Por outro, porque, mesmo se o regime de crescimento for profit-led, é demasiadamente otimista, ou irrealista, imaginar que os efeitos positivos do anúncio de uma política de austeridade sobre as expectativas possam ser suficientemente fortes para contrabalancear os efeitos negativos dos resultados vivenciados e esperados com a recessão. Em resumo, sem crescimento da produtividade e sem demanda capaz de sustentar o crescimento econômico, as políticas de redistribuição de renda acirraram o conflito distributivo no Brasil pós-2011 e contribuiu para acirrar a crise política e econômica.

Por outro lado, a análise não deve se limitar aos resultados dos modelos canônicos. Como já foi mostrado, a inclusão da distribuição interpessoal da renda amplia o leque de possiblidades de investigação e permite uma compreensão mais geral do efeito de determinadas políticas econômicas ${ }^{13}$. É no contexto do modelo ampliado e dos resultados alcançados que entendemos como adequado à realidade brasileira um modelo de desenvolvimento que incorpora os estratos de menor renda, ainda que o padrão de crescimento possa mudar ao longo do tempo. Por padrão de crescimento entende-se a ênfase dada aos componentes da demanda agregada. Com tal opção metodológica, conclui-se, a partir dos resultados obtidos, que a redistribuição interpessoal de renda tem efeitos positivos sobre a acumulação de capital e sobre o grau de utilização da capacidade produtiva, independentemente do regime

\footnotetext{
${ }^{11}$ Em outras palavras, a elasticidade do salário em relação ao volume de emprego é maior do que a elasticidade em relação à taxa real de câmbio.

${ }^{12}$ Nesse caso, vale lembrar que na perspectiva dos modelos neokaleckianos, o papel da oferta tende a ser subestimado.

${ }^{13}$ Ver Carvalho e Rezai, 2015.
} 
de crescimento. Ora, considerando que o Brasil é um dos países mais desiguais do mundo, pode-se inferir que há espaço para ações nesse sentido.

Ao mesmo tempo, reconhece-se que o consumo é, no curto prazo, a variável propulsora que pode dar dinamismo ao crescimento da economia brasileira. Esse parece ter sido o caso ao longo dos 15 anos analisados. Não obstante, entende-se que essa estratégia requer concomitantemente ações complementares direcionadas aos investimentos e às exportações. O fim do boom das commodites, associado à política de valorização cambial, ao contexto internacional e às mudanças repentinas nas políticas econômicas minaram o desempenho desses componentes da demanda agregada. As condições que davam sustentação à elevação do consumo desapareceram. Em outras palavras, ainda que em determinados períodos a redistribuição da renda possa garantir um aumento sustentado do crescimento do consumo e, por conseguinte, do crescimento econômico, é preciso ter em mente as limitações dessa estratégia. Isso porque a expansão do setor de consumo deve ser alinhada com a expansão dos investimentos. Mesmo que a maior demanda do primeiro setor seja capaz de induzir respostas positivas do segundo, deve-se levar em consideração a complexidade que envolve a decisão de investir e as assimetrias que moldam as capacidades de respostas setoriais do investimento. Por exemplo, um aumento da demanda agregada pode sinalizar um aumento de preços e, em contrapartida, um aumento na taxa de juros. Isso pode limitar a expansão dos investimentos produtivos e ampliar os gargalos setoriais.

Logo, o modelo de desenvolvimento requer que a redistribuição de renda esteja associada a uma mudança da estrutura produtiva. Esta exige mais do que políticas de transferência de renda, pois requer coordenação de políticas sociais com políticas macroeconômicas, incluindo preços, juros e câmbio, entre outras. Como já argumentado por Ferrari Filho e Fonseca (2015), para que isso aconteça é necessário alocar a política fiscal e monetária em prol desse objetivo, a começar por mudanças no regime de metas de inflação.

Em síntese, o crescimento econômico pode ser liderado tanto pelos salários como pelas exportações. No curto prazo, o consumo deve ser o protagonista, mas com o passar do tempo as exportações e os investimentos devem ganhar importância. A política macroeconômica, incluindo a política de câmbio real competitivo e de retomada da industrialização, deve estar alinhada com a orientação de longo prazo: crescimento econômico com redistribuição de renda.

\section{CONSIDERAÇÕES FINAIS}

Os resultados do modelo econométrico indicaram que a economia brasileira experimentou um processo de crescimento com características mais próximas de um regime wage-led. As funções "impulso-resposta" das equações estimadas mostraram que um estímulo ao aumento da parcela dos lucros na renda é inconclusivo quando considerada a acumulação de capital. Ademais, foi possível evidenciar que estímulos ao aumento da participação dos lucros na renda e da participação dos 
trabalhadores com altos salários (capitalista-gerentes) no total dos salários têm efeito positivo na poupança agregada, corroborando a hipótese de que a propensão a poupar de indivíduos com altas rendas é mais elevada. O aumento dessa participação tem efeito positivo no aumento da desigualdade total da renda. Por outro lado, dado um aumento da participação da renda dos trabalhadores "comuns" no total dos salários e da participação dos salários no total da renda, tanto a poupança agregada quanto o índice de desigualdade responderam negativamente.

Deve-se considerar, contudo, as limitações do modelo e das variáveis utilizadas. Muitas são as críticas aos modelos de crescimento wage-led, dentre as quais se destaca a que lembra que os modelos não podem ser condição de avaliação para períodos mais longos, tendo em vista que o tipo de crescimento pode variar ao longo do tempo (Foley, 2019). Outro ponto bastante pertinente é que se considera aqui a demanda agregada total, não distinguindo demanda interna e demanda externa, no qual uma economia fechada wage-led não necessariamente será totalmente wage-led quando sujeita à competição internacional (Blecker,1989, 2011). Isso porque, em economias abertas, o efeito do aumento da participação dos salários na renda sobre as transações correntes depende da estrutura da elasticidade-renda das importações e das exportações. Desse modo, o aumento dos salários deve ser acompanhado da elevação do preço dos produtos exportados, caso contrário levará à redução das margens de lucro dos capitalistas, com possível perda de competitividade das exportações (Oreiro et al., 2012).

Sendo assim, argumentou-se em prol da adoção de um modelo de crescimento que seja capaz de conciliar distribuição de renda e políticas de incentivo às exportações. Para tanto, a estratégia de ganhos de participação no comércio internacional deve estar associada à retomada da política industrial, observadas as revoluções tecnológicas e estruturais em curso. Tudo sugere que o Estado deve ser chamado para coordenar os esforços públicos e privados para que a economia aprofunde o adensamento do capital e o progresso tecnológico em meio a todos os setores da sua estrutura produtiva. É possível aproveitar as janelas de oportunidade que se abrem em novos setores como o de energia limpa e renovável e/ou em setores de serviços modernos, associados tanto à indústria como à agricultura, que podem ser complementares e fonte de dinamismo, consideradas as suas disposições setoriais e regionais.

Dito de outro modo, deve-se sempre ter presente que o regime de crescimento é mutável, é um estado, dependendo das condições macroeconômicas nacionais e internacionais vigentes. E é preciso saber identificar e aproveitar as oportunidades, o que significa que a ênfase das políticas conduzidas pelo Estado aos componentes da demanda agregada também deve mudar ao longo do tempo.

\section{REFERÊNCIAS BIBLIOGRÁFICAS}

ARAÚJO, Eliane; GALA, Paulo. (2012) "Regimes de crescimento econômico no Brasil: evidências empíricas e implicações de política”. Estudos Avançados, v. 26, n. 75, p. 41-56. 
BCB. Banco Central do Brasil. (2019). Disponível em:<www.bcb.gov.br>.

BHADURI, A.; S. MARGLIN. (1990) "Unemployment and the real wage: the economic basis for contesting political ideologies". Cambridge Journal of Economics, v. 14, p.375-93.

BLECKER, Robert A. (2011) "Open economy models of distribution and growth". A modern guide to Keynesian macroeconomics and economic policies, p. 215-39.

BLECKER, Robert A (1989) "International competition, income distribution and economic growth". Cambridge Journal of Economics, v.13, p.395-412.

BOWLES, Samuel; BOYER, Robert. (1995 Wages, aggregate demand, and employment in an open economy: an empirical investigation. Macroeconomic policy after the conservative era. Studies in investment, saving and finance. Cambridge: Cambridge University Press.

BRESSER-PEREIRA, L. C.; GALA, P. (2012) "O novo desenvolvimentismo e apontamentos para uma macroeconomia estruturalista do desenvolvimento”. In. OREIRO, J.L; DE PAULA, L.F; BASÍLIO, F. (orgs). Macroeconomia do desenvolvimento: ensaios sobre restrição externa, financiamento $e$ política macroeconômica. Recife: Editora Universitária UFPE.

BRESSER-PEREIRA, L. C.; GALA, P. (2008) "Foreign savings, insufficiency of demand, and low growth.” Journal of Post Keynesian Economics, v. 30, n. 3, pp. 315-334.

CARVALHO, Laura; REZAI, Armon. (2015) "Personal Income Inequality and Aggregate Demand". Cambridge Journal of Economics, v. 40, n. 2, p. 491-505, 2015.

DUTT, Amitava Krishna. (2006) “Aggregate demand, aggregate supply and economic growth".International Review of Applied Economics, v. 20, n. 3, p. 319-336.

DUTT, Amitava Krishna. (1997) “The pattern of direct foreign investment and economic growth". World Development, v. 25, n. 11, p. 1925-1936.

DUTT, Amitava Krishna. (1984) “Stagnation, Income Distribution and Monopoly Power". Cambridge Journal of Economics, v. 8, p. 25-40.

FERRARI FILHO, Fernando; FONSECA, Pedro Cezar Dutra. (2015) "Which developmentalism? A Keynesian-Institutionalist proposal”. Review of Keynesian Economics, v. 3, n. 1, p. 90-107.

FOLEY, Duncan K. (2019) Growth and distribution. Cambridge: Harvard University Press.

FREITAS, Fabio; DWECK, Esther. (2013) "The pattern of economic growth of the Brazilian economy 1970-2005: a demand-led growth perspective”. In: LEVRERO, E.; PALUMBO, Antonella; STIRATI, Antonella (Ed.). Sraffa and the Reconstruction of Economic Theory: Volume Two. Palgrave Macmillan, London, p. 158-191.

FEIJÓ, C. A.; LAMONICA, M. T.; BASTOS, J. C. A. (2015) "Accumulation pattern of the Brazilian economy in the 1990s and 2000s". International Review of Applied Economics, v. 29, n. 1, p. 15-31.

GONÇALVES, J. B. (2017) Distribuição de renda e demanda agregada no Brasil (1995-2013): uma análise a partir de extensões aos modelos neokaleckianos. Dissertação de Mestrado. Universidade de São Paulo.

HEIN, Eckhard; KRÄMER, Hagen. (1997) "Income shares and capital formation: patterns of recent developments". Journal of income distribution, v. 7, n. 1, p. 5-28, 1997.

HEIN, E.; VOGEL, L. (2008) "Distribution and growth reconsidered - empirical results for six OECD countries". Cambridge Journal of Economics, v. 32, p. 479-511.

HOFFMANN, Rodolfo. (2013) “Transferências de renda e desigualdade no Brasil (1995-2011)". Programa Bolsa Família: uma década de inclusão e cidadania. Brasília: IPEA, p. 207-216, 2013.

JETIN, B.; KURT, O. (2011) "Functional Income Distribution and Growth in Thailand: Single Equation Estimations Based on Bhaduri/Marglin Model”. Annual Conference of the Research Network Macroeconomics and Macroeconomic Policies, Berlin,.

LAVOIE, Marc. (1996) "Mark-up pricing versus normal cost pricing in post-Keynesian models". Review of Political Economy, v. 8, n. 1, p. 57-66.

LAVOIE, Marc. (2017) “The origins and evolution of the debate on wage-led and profit-led regimes". European Journal of Economics and Economic Policies: Intervention, v. 14, n. 2, p. 200-221.

LAVOIE, Marc; STOCKHAMMER, Engelbert. (2013) "Wage-led growth: Concept, theories and poli- 
cies”. In: LAVOIE, Marc; STOCKHAMMER, Engelbert (Edit.) Wage-led Growth: An Equitable Strategy for Economic Recovery. Palgrave Macmillan, London, p. 13-39.

MCCOMBIE, John; THIRLWALL, Anthony P. (1994) Economic growth and the balance-of-payments constraint. Springer.

MOLERO, Simarro R. (2011) Functional Distribution of Income and Economic Growth in the Chinese Economy, 1978-2007. School of Oriental and African Studies, Department of Economics Working Papers, n.168, 2011.

MORGAN, Marc. (2017) "Falling Inequality beneath Extreme and Persistent Concentration: New Evidence for Brazil Combining National Accounts, Surveys and Fiscal Data, 2001-2015”. WID -World Working Paper Series. n. 2017/12, 2017. Disponível em: <https://wid.world/document/ extreme-persistent-inequality-new-evidence-brazil-combining-national-accounts-surveys-fiscaldata-2001-2015-wid-world-working-paper-201712/> Acesso em: 12 jul. 2018.

NAASTEPAD, C.W.M., and S. STORM. (2007) "OECD demand regimes (1960-2000)". Journal of Post-Keynesian Economics, v. 29, p.213-248.

ONARAN, Özlem; GALANIS, Giorgos. (2011) "Is aggregate demand wage-led or profit-led? National and global effects". Conditions of Work and Employment Branch, n. 31.

ONARAN, Özlem; STOCKHAMMER, Engelbert. (2005) "Two different export-oriented growth strategies: accumulation and distribution a la Turca and a la South Korea". Emerging Markets Finance and Trade, v. 41 (1), 2005, p. 65-89.

ONARAN, Özlem; STOCKHAMMER; Engelbert, GRAFL, L. (2011) “The finance-dominated growth regime, distribution, and aggregate demand in the US". Cambridge Journal of Economics, v. 35 (4), p. 637-661.

OREIRO, José Luis. L.; PUNZO, L. F.; ARAUJO, Eliane. (2012) "Macroeconomic constraints to growth of the Brazilian economy: diagnosis and some policy proposals". Cambridge Journal of Economics, v. 36, n. 4, p. 919-939.

OREIRO, L. J.; ARAUJO, E. (2013) "Exchange rate misalignment, capital accumulation and income distribution: theory and evidence from the case of Brazil". Panoeconomicus, v. 60, n. 3, p. 381 396.

PALLEY, Thomas I. (2016) "Wage-vs. profit-led growth: the role of the distribution of wages in determining regime character”. Cambridge Journal of Economics, v. 41, n. 1, p. 49-61.

PALLEY, Thomas I. (2014) "Enriching the neo-Kaleckian growth model: nonlinearities, political economy, and theory”.. In. DULLIEN, S., HEIN, E.; TRUGER, A. (eds.), Macroeconomics, Development and Economic Policies: Festschrift for Jan Priewe, Marburg, Metropolis-Verlag, p. 71-84.

PIKETTY, Thomas. (2014) O capital no século XXI. $1^{\text {a }}$ Edição. Rio de Janeiro: Intrínseca.

ROWTHORN, R. (1981) "Demand, Real Wages and Economic Growth". Studi Economici, v. 18, n. 1, p. 3-54.

RUGITSKY, Fernando. (2016) "Milagre, miragem, antimilagre: A economia política dos governos Lula e as raízes da crise atual.” Revista Fevereiro, Dossiê Brasil.

SANTOS, Cláudio Hamilton Matos dos; MODENESI, André de Melo; SQUEFF, Gabriel; VASCONCELOS, Lucas; MORA, Monica; FERNANDES, Thais; MORAES, Thiago; SUMMA, Ricardo; BRAGA, Julia. (2015) "Revisitando a dinâmica trimestral do investimento no Brasil: 1996-2012”. Texto para Discussão IE-UFRJ, n. 05.

SCHETTINI, Bernardo Patta; SANTOS, Cláudio Hamilton Matos dos; AMITRANO, Cláudio Roberto; SQUEFF, Gabriel Coelho; RIBEIRO, Márcio Bruno; GOUVÊA, Raphael Rocha; ORAIR, Rodrigo Octávio; MARTINEZ, Thiago Sevilhano. (2012) "New empirical evidence on the quarterly dynamics of Brazilian aggregate household consumption from 1995 to 2009”. Economia e Sociedade, v. 21, n. 3, p. 607-641

SERRANO, Franklin; SUMMA, Ricardo. (2015) "Aggregate demand and the slowdown of Brazilian economic growth in 2011-2014”. Nova Economia, v. 25, n. spe, p. 803-833.

SERRANO, Franklin; SUMMA, Ricardo. (2012) "Macroeconomic policy, growth and income distribution in the Brazilian economy in the 2000s." Investigación Económica, p. 55-92.

SILVA DE JESUS, C. S.; ARAUJO, R. A.; DRUMOND, C. E. (2018) "An empirical test of the Post- 
Keynesian growth model applied to functional income distribution and the growth regime in Brazil". International Review of Applied Economics, v. 32, n. 4, p. 428-449.

SOUZA, P.H. F. (2017) “A History of Inequality: Top Incomes in Brazil, 1926-2015”. International Policy Centre for Inclusive Growth (IPC-IG), Working Paper, n. 167.

STOCKHAMMER, Engelbert; STEHRER, Robert. (2011) "Goodwin or Kalecki in demand? Functional income distribution and aggregate demand in the short run". Review of Radical Political Economics, v. 43, n. 4, p. 506-522.

STOCKHAMMER, Engelbert.; HEIN, E.; L. GRAFL. (2011) "Globalization and the effects of changes in functional income distribution on aggregate demand in Germany". International Review of Applied Economics, v. 25 (1), p. 1-23.

STOCKHAMMER, Engelbert; ONARAN, Ozlem. (2004) "Wage-led growth: theory, evidence, policy". Review of Keynesian Economics, v. 1, n. 1, p. 61-78.

THIRLWALL, Anthony P. (1979) "The balance of payments constraint as an explanation of the international growth rate diferences”. PSL Quarterly Review, v. 32, n. 128.

TOMIO, B. T. (2016) “Understanding the Brazilian demand regime: a Kaleckian approach". Institute for International Political Economy, Berlin. (Working Paper, n. 73/2016)

VOITCHOVSKY, Sarah. (2005) "Does the profile of income inequality matter for economic growth?" Journal of Economic Growth, v. 10, n. 3, p. 273-296. 